\title{
Airborne Wind Energy Based on Dual Airfoils
}

\author{
Mario Zanon, Sébastien Gros, Joel Andersson and Moritz Diehl
}

\begin{abstract}
The Airborne Wind Energy paradigm proposes to generate energy by flying a tethered airfoil across the wind flow at a high velocity. While Airborne Wind Energy enables flight in higher-altitude, stronger wind layers, the extra drag generated by the tether motion imposes a significant limit to the overall system efficiency. To address this issue, two airfoils with a shared tether can reduce overall system drag. While this technique may improve the efficiency of AWE systems, such improvement can only be achieved through properly balancing the system trajectories and parameters. This paper tackles that problem using optimal control. A generic procedure for modeling multiple-airfoil systems with equations of minimal complexity is proposed. A parametric study shows that at small and medium scales, dual-airfoil systems are significantly more efficient than single airfoil systems, but they are less advantageous at very large scales.
\end{abstract}

Index Terms-Airborne wind energy, dual airfoil, power optimization, large-scale optimization

\section{INTRODUCTION}

To overcome the major difficulties posed by the growing size and mass of conventional wind turbine generators [16], [5], the Airborne Wind Energy (AWE) paradigm proposes to eliminate the structural elements not directly involved in power generation. An emerging consensus recognizes crosswind flight as the most efficient approach to Airborne Wind Energy [17]. Crosswind flight extracts power from the airflow by flying an airfoil tethered to the ground at a high velocity across the wind direction. Power can be generated by (a) performing a cyclical variation of the tether length, together with cyclical variation of the tether tension or (b) by using onboard turbines, transmitting the power to the ground via the tether. In this paper, option (b) is considered, as investigated by e.g. Makani Power [18].

Because it involves a much lighter structure, a major advantage of power generation based on crosswind flight over conventional wind turbines is that higher altitude can be reached and a larger swept area be achieved, thereby reaching wind resources that cannot be tapped into by conventional wind turbines [11].

Unfortunately, the drag due to the motion of the tether during crosswind flight has a significant impact on the system performance. To tackle this issue, the dual-airfoil design was first introduced in [21] and later investigated in e.g. [15], [22], [25]. The key idea of the dual-airfoil desing is to fly two airfoils connected on a single main tether (see Figure 1) in a balanced manner. As a result, only the shorter secondary tethers move at a high velocity and generate drag, while the motion of the main tether is negligible.

M. Zanon, S. Gros, J. Andersson and M. Diehl are with the Elec. Eng. Dept. (ESAT-SCD) and the Optimization in Engineering Center (OPTEC), K.U. Leuven, Kasteelpark Arenberg 10, B-3001 Leuven-Heverlee, Belgium. Corresponding author: sgros@esat.kuleuven.be

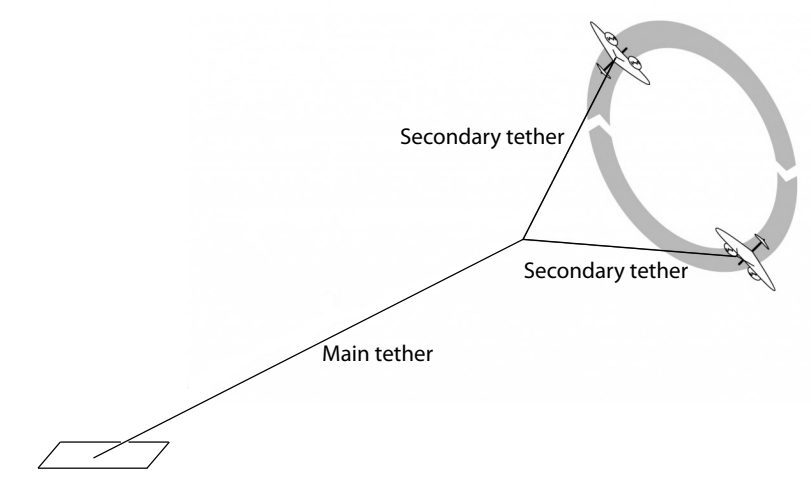

Fig. 1. Schematic of a dual-airfoil AWE system (cf. [21], Fig. 3).

While the dual-airfoil design has the potential to reduce the problem of tether drag for AWE systems, the system design and trajectory must be carefully selected so as to fully exploit the gains of reducing the tether drag. More precisely, a) the airfoil trajectories must balance the forces on the main tether so as to minimize its motion, maintain the optimal airfoil velocities, and maintain an optimal angle between the secondary tethers, b) the aerodynamic forces yielded by onboard power generation must be appropriately chosen so as to maximize the system efficiency, c) the tether lengths must be chosen so as to achieve the best trade-off between reaching higher altitude and adding airborne mass, and d) the tether diameters must be selected so as to achieve the best trade-off between reducing the drag and withstanding the forces in the system.

Defining the optimal system parameters and trajectory is a highly involved problem that is best cast in the framework of optimal control. Single and multiple kite models have been proposed in the literature, see e.g. [14], [15], [24], [7], [12], [13]. This paper, however, proposes a generic modeling procedure for multiple-airfoil AWE systems, including a Finite Element Model (FEM) for the tethers, that is well suited for optimal control and that produces model equations of minimal complexity, so as to reduce the computational burden of evaluating the model sensitivities. The resulting model has 41 states for the single airfoil and 207 states for the dual airfoils. A parametric study of the performance of a dualairfoil system versus a single-airfoil system is presented.

This paper is organized as follows. First a generic modeling procedure for multiple-airfoil systems is proposed and discussed in Section II. Section III describes the powergeneration optimization problem, the solution approach used to compute power-generating trajectories, and the software used to perform the optimization. Section IV proposes a comparison between optimal power generation based on single and dual airfoils for different system scales. Finally, Section 


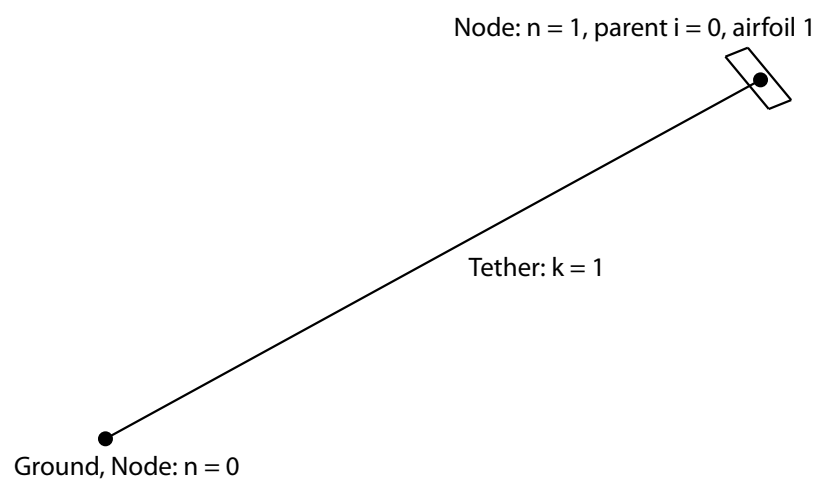

Fig. 2. Schematic of the single-airfoil architecture, with $N=1, \mathcal{A}=\{1\}$, $\mathcal{F}(1)=0$.

$\mathrm{V}$ concludes the paper and outlines further developments.

Contributions of the paper: a generic modeling procedure of minimal computational complexity for multiple-airfoil systems is developed. A large-scale model of single and dual airfoils is developed, including a FEM of the tethers. An optimization procedure to determine the optimal trajectories and design parameters is proposed. A comparison of a dual-airfoil vs. a single-airfoil AWE system is presented.

\section{SySTEM MODEL}

The airfoils are inertially modeled as point-masses. An orthonormal right-handed reference frame $e=\left\{e_{x}, e_{y}, e_{z}\right\}$ attached to the ground is chosen to generate the Cartesian coordinate system defining the positions of the airfoils. The frame $e$ is chosen s.t. a) the wind is blowing in the $e_{x}$-direction, b) the vector $e_{z}$ is opposed to the gravitational acceleration vector $g$. The origin of the coordinate system coincides with the attachment point of the main tether to the ground. In the following, a general procedure for the modeling of multipleairfoil systems is proposed. Both single and dual airfoils are special cases of this formulation, as shown in Figures 2 and 3.

\section{A. System Architecture}

The system is described as a set of $N$ nodes $n \in\{0, \ldots, N\}$ with associated coordinate vectors $X_{n} \in \mathbb{R}^{3}$. The fixed node $X_{0}=[0,0,0]^{T}$ stands for the attachment point of the AWE system to the ground. The subset $\mathcal{A} \subset\{1, \ldots, N\}$ of the set of nodes describes the nodes associated to the airfoils. Assuming a tree structure, to each node $n \in\{1, \ldots, N\}$ a single tether $k=n$ is associated, and the parent node $i$ to which the tether is attached is defined by the map $i=\mathcal{F}(n)$. See Figures 2 and 3 for an illustration. The system architecture is then defined by the number of nodes $N$, the set $\mathcal{A}$, and the map $\mathcal{F}$. Note that the proposed formulation allows for tree-like system architectures only.

In the following, the component-wise notation $X_{n}=$ $\left[x_{n}, y_{n}, z_{n}\right]^{T}$ of the node coordinate vectors $X_{n}$ is used. The position of the node $n$ is given by $\mathcal{P}_{n}=x_{n} e_{x}+y_{n} e_{y}+z_{n} e_{z}$. Each tether $k=1, \ldots, N$ has associated length $l_{k}$ and diameter $d_{k}$.

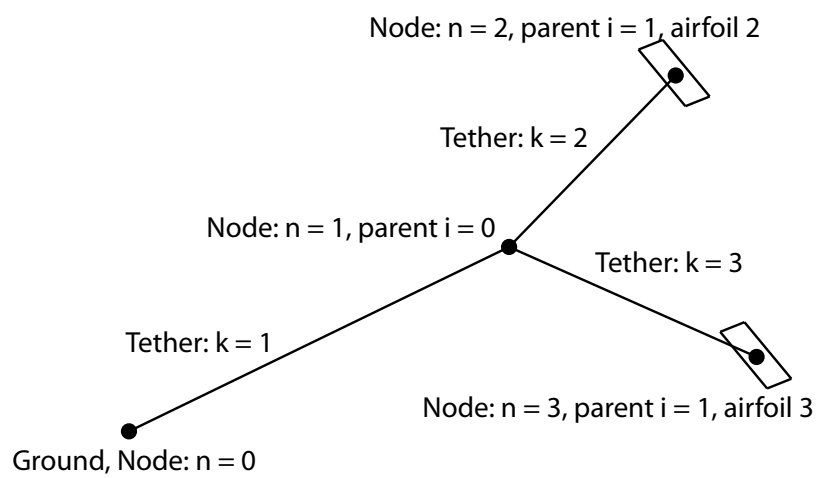

Fig. 3. Schematic of the dual-airfoil architecture, with $N=3, \mathcal{A}=\{2,3\}$, $\mathcal{F}(1)=0, \mathcal{F}(2)=1, \mathcal{F}(3)=1$.

\section{B. Airfoil model}

For any node $n \in\{0, \ldots, N\}$, we define the velocity relative to the airmass:

$$
v_{n}=\left(\dot{x}_{n}-W\right) e_{x}+\dot{y}_{n} e_{y}+\dot{z}_{n} e_{z},
$$

where $W \in \mathbb{R}$ is the local wind velocity in the $e_{x}$ direction. A generalization of this formulation to a $3 \mathrm{D}$ wind field is straightforward. If $n \in \mathcal{A}$, the norms of the lift and drag forces acting on the airfoil $n$ are given by [20]:

$$
\left\|F_{\mathrm{L}}^{n}\right\|=\frac{1}{2} \rho S C_{\mathrm{L}}^{n}\left\|v_{n}\right\|^{2}, \quad\left\|F_{\mathrm{D}}^{n}\right\|=\frac{1}{2} \rho S C_{\mathrm{D}}^{n}\left\|v_{n}\right\|^{2},
$$

where $C_{\mathrm{L}}^{n}$ and $C_{\mathrm{D}}^{n}$ are the lift and drag coefficients of the airfoil, $\rho$ is the air density and $S$ the airfoil surface.

The lift force is defined to be orthogonal to the relative velocities $v_{n}$ of the airfoil. Moreover, it is assumed in this model that the lift force is orthogonal to the airfoil transversal axis [20], [8]. Airfoil $n$ is linked to its parent node $i=\mathcal{F}(n)$ by tether $n$. One can form the unitary coordinate vector:

$$
e_{r}^{n}=\frac{X_{n}-X_{i}}{\left\|X_{n}-X_{i}\right\|}, \quad i=\mathcal{F}(n)
$$

and introduce the following definition of the transversal and lift axis:

$$
\begin{aligned}
e_{T}^{n} & =\frac{v_{n} \times e_{r}^{n}}{\left\|v_{n} \times e_{r}^{n}\right\|}, \\
f_{L}^{n} & =e_{T}^{n} \times v_{n} .
\end{aligned}
$$

It can be observed that vector $e_{T}^{n}$ is normed to 1 , thus vector $f_{L}^{n}$ is normed to $\left\|v_{n}\right\|$. Because $f_{L}^{n}$ is orthogonal to the relative velocity $v_{n}$ and lies in the plane spanned by $\left\{e_{r}^{n}, v_{n}\right\}$, if the airfoil is not tilted with respect to vector $e_{r}^{n}$, the lift force acts along vector $f_{L}^{n}$. Introducing the roll angle $\psi_{i}$ describing the tilting of the lift force around the axis $v_{n}$, the lift force can be defined by:

$$
F_{\mathrm{L}}^{n}=\frac{1}{2} \rho S C_{\mathrm{L}}^{n}\left(\cos \left(\psi_{i}\right) f_{L}^{n}\left\|v_{n}\right\|-\sin \left(\psi_{i}\right) e_{T}^{n}\left\|v_{n}\right\|^{2}\right) .
$$

By definition $F_{\mathrm{L}}^{n}$ is always orthogonal to $v_{n}$, and lies in the plane defined by $\left\{e_{r}^{n}, v_{n}\right\}$ if $\psi_{i}=0$.

The airfoil drag force is opposed to the relative velocity, and is readily given by:

$$
F_{\mathrm{D}}^{n}=-\frac{1}{2} \rho S C_{\mathrm{D}}^{n}\left\|v_{n}\right\| v_{n}
$$


The drag generated by the onboard turbines can be modeled as:

$$
F_{G}^{n}=-\kappa_{n}\left\|v_{n}\right\| v_{n},
$$

where $\dot{\kappa}_{n}(t)=u_{\kappa_{n}(t)}, u_{\kappa_{n}(t)}$ is a control variable and we assume that the generated force is opposed to the relative velocity. The resulting aerodynamic power is:

$$
P_{n}=v_{n}^{T} F_{G}^{n}=-\kappa_{n}\left\|v_{n}\right\|^{3} .
$$

The resulting airfoil aerodynamic force acting on airfoil $n$ is given by $F_{\mathrm{A}}^{n}=F_{\mathrm{L}}^{n}+F_{\mathrm{D}}^{n}+F_{G}^{n}$.

In this model, it is assumed that the time-derivative of the lift coefficient is directly controlled, and the drag coefficient $C_{\mathrm{D}}^{n}$ is approximated by [20], [8]:

$$
C_{\mathrm{D}}^{n}=C_{\mathrm{D}}^{0}+C_{\mathrm{D}}^{I}\left(C_{\mathrm{L}}^{n}\right)^{2},
$$

where $C_{\mathrm{D}}^{0}$ and $C_{\mathrm{D}}^{I}$ are the airfoil drag and induced-drag coefficients respectively.

The kinetic and potential energy functions associated with the airfoil dynamics are:

$$
\mathcal{T}_{\mathrm{A}}^{n}=\frac{1}{2} M_{\mathrm{A}}\left\|\dot{X}_{n}\right\|^{2}, \quad \nu_{\mathrm{A}}^{n}=M_{\mathrm{A}} g z_{n},
$$

where $M_{\mathrm{A}}$ is the airfoil mass, and the Lagrange function for the airfoils reads:

$$
\mathcal{L}_{\mathrm{A}}=\mathcal{T}_{\mathrm{A}}-\mathcal{V}_{\mathrm{A}}, \quad \mathcal{T}_{\mathrm{A}}=\sum_{n \in \mathcal{A}} \mathcal{T}_{\mathrm{A}}^{n}, \quad \mathcal{V}_{\mathrm{A}}=\sum_{n \in \mathcal{A}} \mathcal{V}_{\mathrm{A}}^{n} .
$$

\section{Wind and atmosphere model}

Assuming a laminar wind flow with a logarithmic wind shear model blowing uniformly in the $e_{x}$-direction, the freeflow windspeed $W_{\infty}$ at altitude $z$ is given by [19]:

$$
W_{\infty}(z)=W_{0} \frac{\log \left(z / z_{\mathrm{r}}\right)}{\log \left(z_{0} / z_{\mathrm{r}}\right)},
$$

where $W_{0} \in \mathbb{R}$ is the wind velocity at altitude $z_{0}$ and $z_{r}$ is the ground roughness.

To account for the drop of density with altitude the following atmospheric model is introduced [2]:

$$
\begin{aligned}
& T(z)=T_{0}-T_{L} z, \\
& P(z)=P_{0}\left(1-\frac{T_{L} z}{T_{0}}\right)^{\frac{g M_{a}}{R T_{L}},}, \\
& \rho(z)=\frac{P M_{a}}{R T},
\end{aligned}
$$

where $T_{0}$ is the sea level standard temperature, $T_{L}$ is the temperature lapse rate, $P_{0}$ is the pressure at sea level, $M_{a}$ is the molar air density and $R$ is the universal gas constant.

\section{Tether model}

In the proposed formulation, the tethers are modeled with a lumped mass Finite Element Model. For a rigid tether $k \in\{1, \ldots, N\}$ of length $l_{k}$, density $\rho_{c}$, diameter $d_{k}$, we define $N_{k}$ elements linked by massless rigid links, where link $k, j$ connects elements $k, j$ and $k, j+1$. Note that with this notation, the position of the endpoint $X_{k, N_{k}}$ of each tether $k$ coincides with the position $X_{k}$ of node $k$. The index $j$ ranges from 1 to $N_{k}$ when it refers to the elements and from 1 to $N_{k}-1$ when it refers to the links between elements. In the proposed model, all links have the same length $l_{k, j}=l_{k} /\left(N_{k}-1\right)$ and each element $k, j$ with $2<j<N_{k}$ has mass $m_{k, j}=m_{k} /\left(N_{k}-1\right)$, while $m_{k, 1}=m_{k, N_{k}}=m_{k} /\left(2\left(N_{k}-1\right)\right)$. The tether kinetic and potential energy functions read:

$$
\begin{aligned}
\mathcal{T}_{\mathrm{T}}^{k} & =\sum_{j=1}^{N_{k}} \frac{1}{2} m_{k, j}\left\|\dot{X}_{k, j}\right\|^{2}, \\
\mathcal{V}_{\mathrm{T}}^{k} & =\sum_{j=1}^{N_{k}} m_{k, j} g z_{k, j},
\end{aligned}
$$

where $m_{k, j}$ is the mass associated with each element and $\dot{X}_{k, j}$ and $z_{k, j}$ are respectively its velocity and height.

The tether drag on each tether section $k, j$ is given by:

$$
F_{S}^{k, j}=-\frac{1}{2} \rho_{k, j} d_{k} l_{k, j} C_{\mathrm{T}}\left\|v_{k, j}\right\| v_{k, j}
$$

where $C_{\mathrm{T}}$ is the drag coefficient of a cylinder, $l_{k, j}$ is the length of link $k, j$ and $v_{k, j}$ is the velocity of its midpoint, computed as

$$
v_{k, j}=\frac{\dot{X}_{k, j}+\dot{X}_{k, j+1}}{2}-W\left(\frac{z_{k, j}+z_{k, j+1}}{2}\right),
$$

where $W$ is the windspeed at the midpoint's altitude. The lift generated by the tethers is not considered in this formulation. The contribution of the tether drag forces to the generalized forces acting on the generalized coordinates $X_{k, j}$ is given by:

$$
F_{T}^{k, j}=\frac{F_{S}^{k, j}+F_{S}^{k, j+1}}{2} .
$$

\section{E. Generalized forces}

The vector of generalized forces $F=\left[F_{1,1}^{T}, \ldots, F_{N, N_{N}}^{T}\right]^{T}$, where $F_{k, j} \in \mathbb{R}^{3}$ is the vector of generalized forces acting on the vector of generalized coordinates $X_{k, j}$, is resulting from the sum of the various contributions coming from tether drags and airfoil aerodynamic forces. Though this summation can be performed very intuitively, it can be formulated as the following systematic construction. For any $k \in\{1, \ldots, N\}, j \in\left\{1, \ldots, N_{k}\right\}$, $F_{k, j}$ is given by:

$$
F_{k, j}=\left\{\begin{array}{lll}
F_{T}^{1,1} & \text { if } \quad j=1, k=1 \\
F_{T}^{k, j} & \text { if } \quad j \in 2, \ldots, N_{k}-1 \\
F_{T}^{k, j}+\sum F_{T}^{k_{c}, 1} & \text { if } \quad \mathcal{F}\left(k_{c}\right)=k \text { and } j=N_{k} \\
F_{T}^{k, j}+F_{\mathrm{A}}^{k} & \text { if } \quad k \in A \text { and } j=N_{k}
\end{array}\right.
$$

\section{F. System model}

In the following, the generalized coordinate vector $X=$ $\left[X_{1,1}^{T}, \ldots, X_{N, N_{N}}^{T}\right]^{T}$ of the system is used. The system is considered as a set of independent tethers and airfoils, with associated Lagrange functions. The tethers introduce a set of constraints in the system configuration, given by:

$$
G_{k, j}=\frac{1}{2}\left(\left(X_{k, j+1}-X_{k, j}\right)^{T}\left(X_{k, j+1}-X_{k, j}\right)-l_{k, j}^{2}\right)=0,
$$


for $k=1, \ldots, N, j=1, \ldots, N_{k}$. The system Lagrange function reads:

$$
\mathcal{L}=\mathcal{T}-\mathcal{V}-\lambda^{T} G, \quad \mathcal{T}=\mathcal{T}_{\mathrm{A}}+\mathcal{T}_{\mathrm{T}}, \quad \mathcal{V}=\mathcal{V}_{\mathrm{A}}+\mathcal{V}_{\mathrm{T}},
$$

where $\lambda \in \mathbb{R}^{K}$ is the vector of Lagrange multipliers associated to the constraints $G$. Using the Lagrange equation [9] $\frac{d}{d t} \frac{\partial \mathcal{L}}{\partial \dot{X}}-$ $\frac{\partial \mathcal{L}}{\partial X}=F$, it can be verified that the system dynamics are given by the following index-3 DAEs:

$$
\mathcal{T}_{\dot{X} \dot{X}} \ddot{X}+G_{X}^{T} \lambda+\mathcal{V}_{X}=F, \quad G=0,
$$

where $\lambda$ is the DAE algebraic state, $G_{X}=\frac{\partial G}{\partial X}, \mathcal{T}_{\dot{X} \dot{X}}=\frac{\partial^{2} \mathcal{T}}{\partial \dot{X}^{2}}$ and $\nu_{X}=\frac{\partial \nu}{\partial X}$.

For any $t_{0} \in \mathbb{R}$, equation (3) can be reformulated as an index1 DAE by performing index reduction, which yields $\ddot{G}(t)=$ $0, \dot{G}\left(t_{0}\right)=0, G\left(t_{0}\right)=0$. The resulting equations read (together with the consistency conditions):

$$
\begin{aligned}
& {\left[\begin{array}{cc}
\mathcal{T}_{\dot{X} \dot{X}} & G_{X}^{T} \\
G_{X} & 0
\end{array}\right]\left[\begin{array}{c}
\ddot{X} \\
\lambda
\end{array}\right]=\left[\begin{array}{l}
F-\mathcal{V}_{X} \\
-\frac{\partial}{\partial X}\left(G_{X} \dot{X}\right) \dot{X}
\end{array}\right],} \\
& G\left(t_{0}\right)=0, \quad \dot{G}\left(t_{0}\right)=\left(G_{X} \dot{X}\right)_{t=t_{0}}=0 .
\end{aligned}
$$

It can be verified that the tension in tether $k$ is readily given by:

$$
\Gamma_{k}=\lambda_{k} l_{k}
$$

For long integration times, a correction of the numerical drift of $G$ may be required.

Note that (4) can be treated as an ODE by inverting the DAE mass matrix so as to compute $\ddot{X}$ and $\lambda$ explicitly. While this approach sounds appealing, and can be efficient for model simulations if the mass matrix is inverted numerically, the symbolic expressions for the resulting ODE are highly complex and the sparsity in the model expressions is usually lost. As a result, computing the model sensitivities given in its ODE formulation is very expensive. In the framework of optimization, the system model is therefore best treated in the implicit form (4), using implicit integration methods.

1) Dual-airfoil model: the system architecture reads (see Figure 3):

$N=3, \quad \mathcal{A}=\{2,3\}, \quad \mathcal{F}(1)=0, \quad \mathcal{F}(2)=1, \quad \mathcal{F}(3)=1$,

the coordinate vector is $X \in \mathbb{R}^{3\left(N_{1}+N_{2}+N_{3}\right)}$ and the constraints are defined by:

$$
G=\frac{1}{2}\left[\begin{array}{c}
\left(\left(X_{1,2}-X_{1,1}\right)^{T}\left(X_{1,2}-X_{1,1}\right)-l_{1,1}^{2}\right) \\
\vdots \\
\left(\left(X_{1, N_{1}}-X_{1, N_{1}-1}\right)^{T}\left(X_{1, N_{1}}-X_{1, N_{1}-1}\right)-l_{1, N_{1}-1}^{2}\right) \\
\left(\left(X_{2,2}-X_{2,1}\right)^{T}\left(X_{2,2}-X_{2,1}\right)-l_{2,1}^{2}\right) \\
\vdots \\
\left(\left(X_{2, N_{2}}-X_{2, N_{2}-1}\right)^{T}\left(X_{2, N_{2}}-X_{2, N_{2}-1}\right)-l_{2, N_{2}-1}^{2}\right) \\
\left(\left(X_{3,2}-X_{3,1}\right)^{T}\left(X_{3,2}-X_{3,1}\right)-l_{3,1}^{2}\right) \\
\vdots \\
\left(\left(X_{3, N_{3}}-X_{3, N_{3}-1}\right)^{T}\left(X_{3, N_{3}}-X_{3, N_{3}-1}\right)-l_{3, N_{3}-1}^{2}\right)
\end{array}\right],
$$

where $X_{1,1}=X_{0}=[0,0,0]^{T}$, the joint position is $X_{1, N_{1}}=$ $X_{2,1}=X_{3,1}=X_{1}$, the first airfoil position is $X_{2, N_{2}}=X_{2}$ and the second airfoil position is $X_{3, N_{3}}=X_{3}$. The two airfoils and the two secondary tethers are considered identical. The discretization is thus also identical and $N_{2}=N_{3}$.

2) Single-airfoil model: the system architecture reads (see Figure 2):

$$
N=1, \quad \mathcal{A}=\{1\}, \quad \mathcal{F}(1)=0,
$$

the coordinate vector is $X \in \mathbb{R}^{3 N_{1}}$ and the constraints are defined by:

$G=\frac{1}{2}\left[\begin{array}{c}\left(\left(X_{1,2}-X_{1,1}\right)^{T}\left(X_{1,2}-X_{1,1}\right)-l_{1,1}^{2}\right) \\ \vdots \\ \left(\left(X_{1, N_{1}}-X_{1, N_{1}-1}\right)^{T}\left(X_{1, N_{1}}-X_{1, N_{1}-1}\right)-l_{1, N_{1}-1}^{2}\right)\end{array}\right]$,

where $X_{1,1}=X_{0}=[0,0,0]^{T}$ and $X_{1, N_{1}}=X_{1}$.

\section{G. Model assumptions \& discussion}

The proposed model is based on the following assumptions:

1) the tethers are modelled with a lumped-mass finite element model

2) the lift forces are orthogonal to the airfoil transversal axis

3) the airfoils have a perfect yaw control, resulting in no side-slip

4) the time-derivatives of the lift coefficient and roll angle are controlled and actuation is instantaneous

5) the time-derivative of the onboard turbine drag coefficient is controlled and actuation is instantaneous

The proposed model construction can straightforwardly accommodate different tether and airfoil models, e.g.: a 6-DOF airfoil description and more elaborate aerodynamic models. Yet in this paper a simple model was preferred, so as to reduce the complexity of the presentation. Further research will seek at improving the tether models by including the tether aerodynamic lift and elasticity.

In this paper, no assumption has been made on the interaction between the airfoils and the airmass. For conventional wind turbines, Betz first developed a simplified model [19], [3]. While such a formulation can be adapted for AWE systems and included in the problem formulation, experimental data is needed to assess the validity of such a simplified model. This validation process is the subject of ongoing research at $\mathrm{KU}$ Leuven.

\section{OPTIMIZATION PROBLEM}

The airfoil trajectories as well as the tether lengths and sections are manipulated so as to maximize the system average power generation over an orbit of free duration $T_{p}$. The periodicity of the system is guaranteed by satisfying the boundary conditions:

$$
X(0)-X\left(T_{p}\right)=0
$$

However, it can be observed that (6) together with (5) form a redundant set of equality constraints, violating the Linear 
Independence Constraint Qualification (LICQ). The directions violating the consistency conditions must then be removed from the set of periodicity conditions [6]. Defining a matrix $Z$ that forms a basis of the null-space of:

$$
J=\left[\begin{array}{l}
\frac{\partial G}{\partial X} \\
\frac{\partial G}{\partial X}
\end{array}\right]_{t=0},
$$

i.e. $J Z=0$, the set of consistency conditions (5) together with:

$$
Z^{T}\left(X(0)-X\left(T_{p}\right)\right)=0,
$$

have no redundancy. The basis $Z$ is non-unique, and can be chosen so as to limit its computational complexity. As an alternative, it can also be introduced as a set of parameters in the optimization algorithm, and computed numerically.

In order to ensure that the tethers are always under tension but that their resistance is never exceeded, the constraints:

$$
\frac{\gamma}{f_{s}} \frac{\pi}{4} d_{k}^{2} \geq \Gamma_{k}(t)=\lambda_{k} l_{k} \geq 0, \quad \forall t, k=1, \ldots, N,
$$

are imposed, where $\gamma$ is the tether yield strength and $f_{s}$ the safety factor. Moreover, the following bounds are proposed:

$$
\begin{aligned}
0 & \leq C_{\mathrm{L}}^{i} \leq 1, & -5 \mathrm{~s}^{-1} \leq \dot{C}_{\mathrm{L}}^{i} \leq 5 \mathrm{~s}^{-1} \\
-80^{\circ} & \leq \psi_{L}^{i} \leq 80^{\circ}, & -5 \mathrm{~s}^{-1} \leq \dot{\psi}_{L}^{i} \leq 5 \mathrm{~s}^{-1} \\
-1000 \mathrm{~kg} /(\mathrm{ms}) & \leq \dot{\kappa}_{i} \leq 1000 \mathrm{~kg} /(\mathrm{ms}), & \forall t, i \in \mathcal{A} .
\end{aligned}
$$

The periodic power optimization problem reads:

$$
\begin{aligned}
\bar{P}=\max _{U, X, \theta, T_{p}} & \frac{1}{T_{p}} \int_{0}^{T_{p}} \sum_{i \in \mathcal{A}} P_{i} d t, \\
\text { s.t. } & (4)-(5),(7)-(9)
\end{aligned}
$$

where $U_{i}=\left\{\dot{C}_{\mathrm{L}}^{i}, \dot{\psi}_{L}^{i}, \kappa_{i}\right\}, i \in \mathcal{A}, \theta_{k}=\left\{l_{k}, d_{k}\right\}, k=1, \ldots, N$. Note that $T_{p}$ is an optimization variable, thus the duration of the orbit will be adapted by the optimizer so as to maximize the average power. In order to be able to treat this problem, a time transformation can be introduced, as proposed in [10, p. 27].

\section{A. Solution approach}

The Optimal Control Problem (10) is large-scale and highly non-convex and therefore requires a good initial guess to be tackled by derivative-based optimization. However, no such guess is readily available. To address this issue, a complex procedure is needed to compute an initial guess for problem (10). For the sake of brevity the details of this procedure will be omitted.

For the dual-airfoil system, solving (10) on a full orbit yields quasi-identical trajectories for the two airfoils, hence (10) was solved on a half orbit instead, using the periodicity conditions $X_{2}(0)=X_{3}\left(\frac{1}{2} T_{p}\right), X_{3}(0)=X_{2}\left(\frac{1}{2} T_{p}\right)$ so as to match the terminal state of one airfoil with the initial state of the other. For both the single and dual-airfoil problems, the control input profiles were discretized using a piecewise-constant parametrization having 20 intervals per full orbit. One collocation element has been used per control interval.
TABLE I

FIXED MODEL PARAMETERS

\begin{tabular}{|c||c|c|c|}
\hline Parameter & Symbol & Value & Unit \\
\hline Air density & $\rho$ & 1.23 & $\mathrm{~kg} \cdot \mathrm{m}^{3}$ \\
Tethers density & $\rho_{c}$ & 1450 & $\mathrm{~kg} \cdot \mathrm{m}^{3}$ \\
Airfoil parasitic drag coefficient & $C_{D}^{0}$ & 0.02 & - \\
Airfoil induced drag coefficient & $C_{D}^{P}$ & 0.02 & - \\
Airfoil aspect ratio & $A_{R}$ & 10 & - \\
Wind velocity at altitude $z_{0}$ & $W_{0}$ & 10 & $\mathrm{~m} / \mathrm{s}$ \\
Altitude of wind velocity $W_{0}$ & $z_{0}$ & 100 & $\mathrm{~m}$ \\
Roughness factor & $z_{r}$ & 0.1 & $\mathrm{~m}$ \\
Sea level standard temperature & $T_{0}$ & 288.15 & $\mathrm{~K}$ \\
Temperature lapse rate & $T_{L}$ & 0.0065 & $\mathrm{~K} / \mathrm{m}$ \\
Sea level pressure & $P_{0}$ & 101325 & $\mathrm{~Pa}$ \\
Molar air density & $M_{a}$ & 0.0289644 & $\mathrm{~kg} / \mathrm{mol}$ \\
Universal gas constant & $R$ & 8.31447 & $\mathrm{~J} /(\mathrm{molK})$ \\
Tether drag coefficient & $C_{\mathrm{T}}$ & 1 & - \\
Tether yield strength & $\gamma$ & $3.9 \cdot 10^{9}$ & $\mathrm{~Pa}$ \\
Safety factor & $f_{s}$ & 5 & - \\
\hline \multicolumn{2}{|c}{} \\
\hline
\end{tabular}

\section{B. Methods \& Software}

Because dynamics (4) are unstable, a simultaneous optimal control technique is required to optimize the system model. In this paper, the discretization of the model dynamics (4) was based on a direct collocation approach [4], where the model simulation, constraints and optimization are handled simultaneously in a large-scale sparse Nonlinear Program (NLP). Collocation approaches provide a straightforward way to deal with implicit index-1 DAE systems [4].

The problem transcription was performed using the opensource optimization framework CasADi [1]. The resulting NLP was solved using the interior-point solver IPOPT 3.10.1 [23] using WSMP as a linear solver.

\section{PARAmetric STUdy}

The parametric studies aim at assessing the relationship between the total airfoil surface and the average generated power, i.e. $\bar{P}\left(S_{\text {tot }}\right)$ where $S_{\text {tot }}=\sum_{n \in \mathcal{A}} S$. This study focused on assessing if, for a given total airfoil surface, a single or a dual-airfoil system should be preferred.

The study was based on airfoils having a maximum gliding ratio $L / D=25$. The tethers are assumed to be made of Dyneema $^{\circledR}$, which has a very high stiffness and yield strength for a low density. The fixed model parameters are summarized in Table I.

For the single airfoil the tether has been discretized using 5 segments. For the dual airfoils the main tether has been discretized using 20 segments, while 5 segments were used for both secondary tethers. This results in 41 states for the single airfoil and 207 states for the dual airfoils. More refined tether discretizations have been tested and the obtained results don't show any relevant difference.

Problem (10) being nonconvex, there is no a priori guarantee that the computed solution is a global optimum. Nevertheless, using insights on the physics of the system, it is possible to assess the solution and compare it to the results of simplified studies, such as the ones proposed in [17]. Initializing problem (10) at different intial guesses, it has been observed that the NLP solver consistently converges to the same solution, hence suggesting that it is the optimum of a reasonably large set of possible trajectories. 

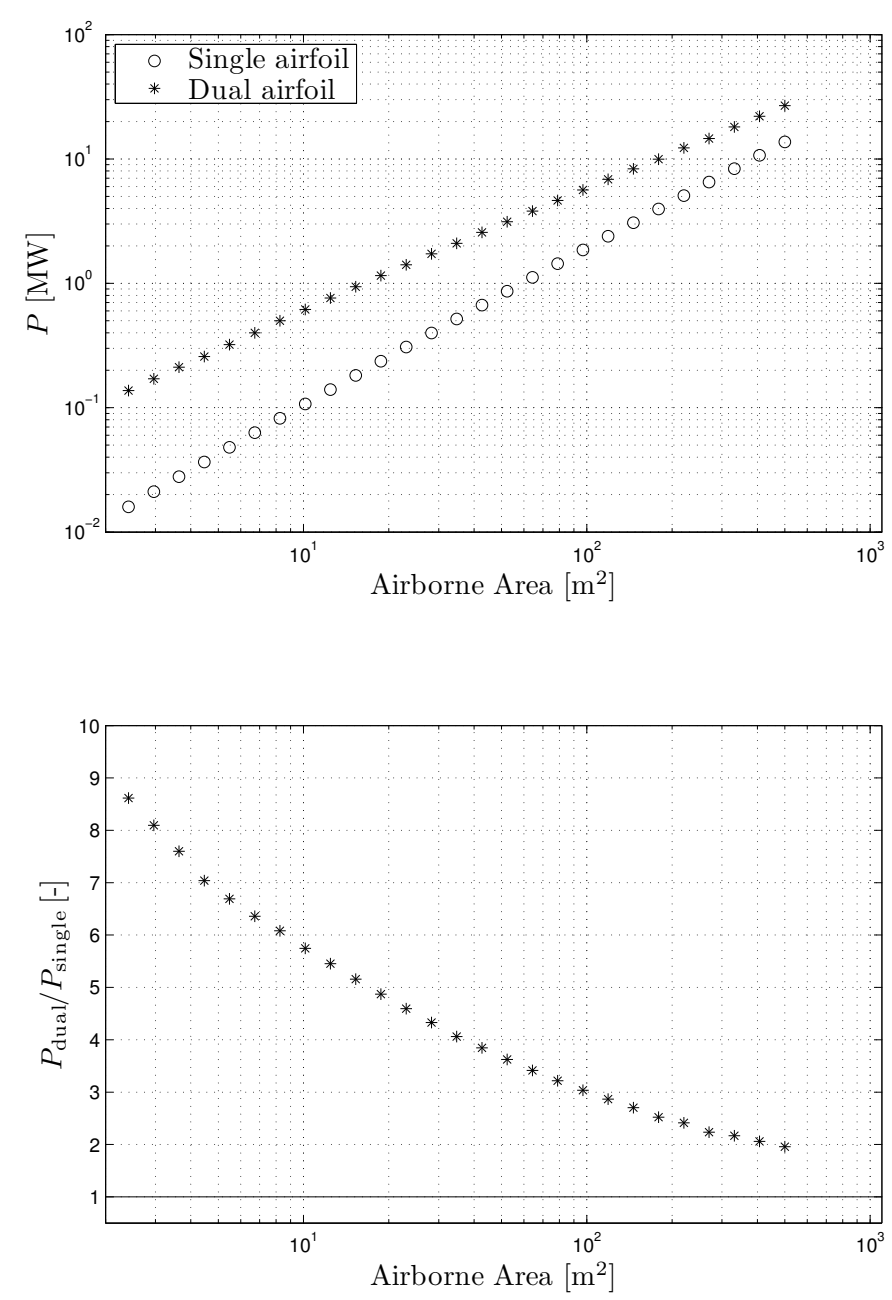

Fig. 4. Dual vs. one large single airfoil: average power output for different wing surface $S$, keeping the wing loading constant. The comparison assumes that both systems have the same overall airfoil surface, i.e. $S_{\text {dual }}=\frac{1}{2} S_{\text {single }}$, hence assessing the advantage of having a dual-airfoil system with two smaller airfoils vs. having a single large airfoil.

Using the method proposed in Subsection III-A a solution to (10) for the single-airfoil system using parameter values $S=500 \mathrm{~m}^{2}$ and $M_{A} / S=20 \mathrm{~kg} / \mathrm{m}^{2}$, and a solution for the dual-airfoil system using parameter values $S=250 \mathrm{~m}^{2}$ and $M_{A} / S=20 \mathrm{~kg} / \mathrm{m}^{2}$ are computed.

Starting from these solutions, a homotopy with respect to the total airfoil area $S_{\text {tot }}$ is applied to (10). Keeping the wing loading $M_{A} / S=20 \mathrm{~kg} / \mathrm{m}^{2}$ constant (i.e. the airfoil mass scales linearly with the total airfoil area), $S_{\text {tot }}$ is gradually reduced and (10) repeatedly solved, using the solution from the previous step as an initial guess for the subsequent step. The average generated power for both the single and dual-airfoil systems are displayed in Figure 4, top graph. The ratio between the average generated power for the dual-airfoil system and the single-airfoil system is displayed in Figure 4, bottom graph. Note that the graphs in Figure 4 display the mechanical power dissipated by the onboard turbines. The actual electrical power depends on the generators and converters efficiency, whose value is arguably similar for the two systems. For the chosen parameters, the dual-airfoil system is always more

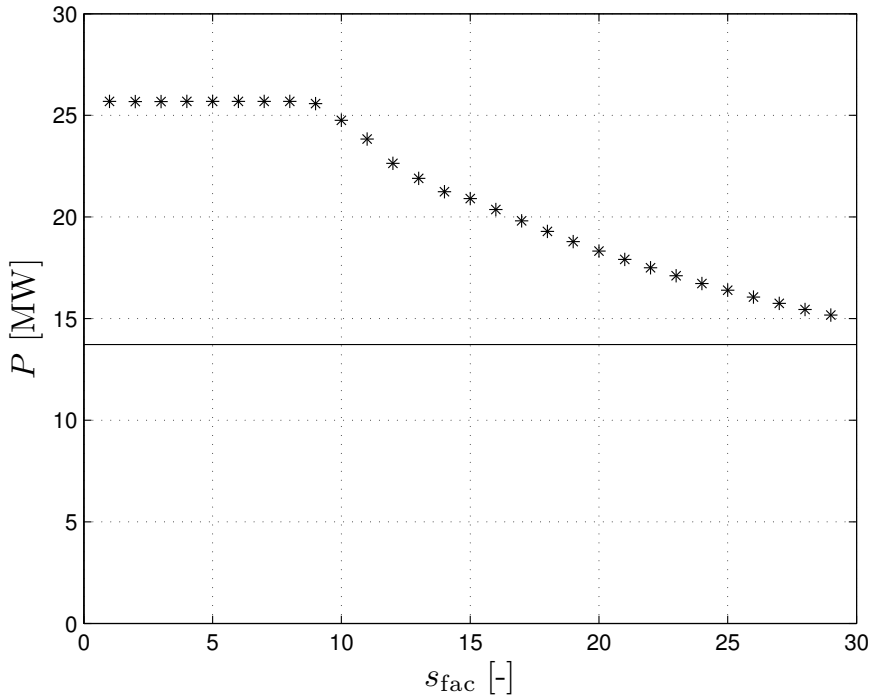

Fig. 5. Study of the impact of the safety constraint on the extracted power $P$ for the largest dual-airfoil system. The parameter $s_{\text {fac }}$ expresses the distance between the airfoils, measured in wingspans. For comparison, the energy extracted by the single airfoil is displayed as a continuous line.

advantageous than the single one. As the total airfoil surface increases, however, the ratio between the power extracted by the dual and single airfoil decreases significantly.

It should be observed that the required total airfoil surface for a desired amount of average generated power is also indirectly assessed through the proposed parametric study. Indeed, it can be seen from Figure 4 that for an average power generation of $10 \mathrm{MW}$ the dual-airfoil system requires a total airfoil surface of approximately half the one of the singleairfoil system, but the dual airfoil system requires gradually less airfoil surface as the desired average generated power decreases.

For safety reasons, it is desirable that the dual-airfoil trajectories keep the airfoils far from each other, thus avoiding the risk of collisions. A second reason for having the airfoils flying large orbits, is to reduce the interaction with the airmass. In this paper no hypothesis is made on this complex interaction, which is assumed to be small and is thus neglected. This assumption might not hold if the airfoils fly too close to each other, as the interaction will be higher. A study has thus been done, to check how the extracted power is affected by imposing a safety constraint on the distance between the airfoils. This safety constraint is expressed as

$$
\left(X_{2}-X_{3}\right)^{T}\left(X_{2}-X_{3}\right) \geq\left(s_{\mathrm{fac}} w_{\mathrm{s}}\right)^{2},
$$

where the wingspan is given by $w_{\mathrm{s}}=\sqrt{S A_{\mathrm{R}}}$, with $A_{\mathrm{R}}$ the aspect ratio. For the simulations, the value $A_{\mathrm{R}}=10$ has been chosen. The results are displayed in Figure 5 for the largest dual-airfoil system, i.e. $S_{\text {tot }}=500 \mathrm{~m}^{2}$. It can be seen that allowing the airfoils to fly closer than $9 w_{\mathrm{s}}$ does not lead to an increase in the extracted power. For larger orbits, the extracted power diminishes but the loss is not dramatic and, even for very large orbits, the dual airfoils still extract more power than the single airfoil.

The trajectory for the biggest systems considered, i.e. $S_{\text {tot }}=$ 


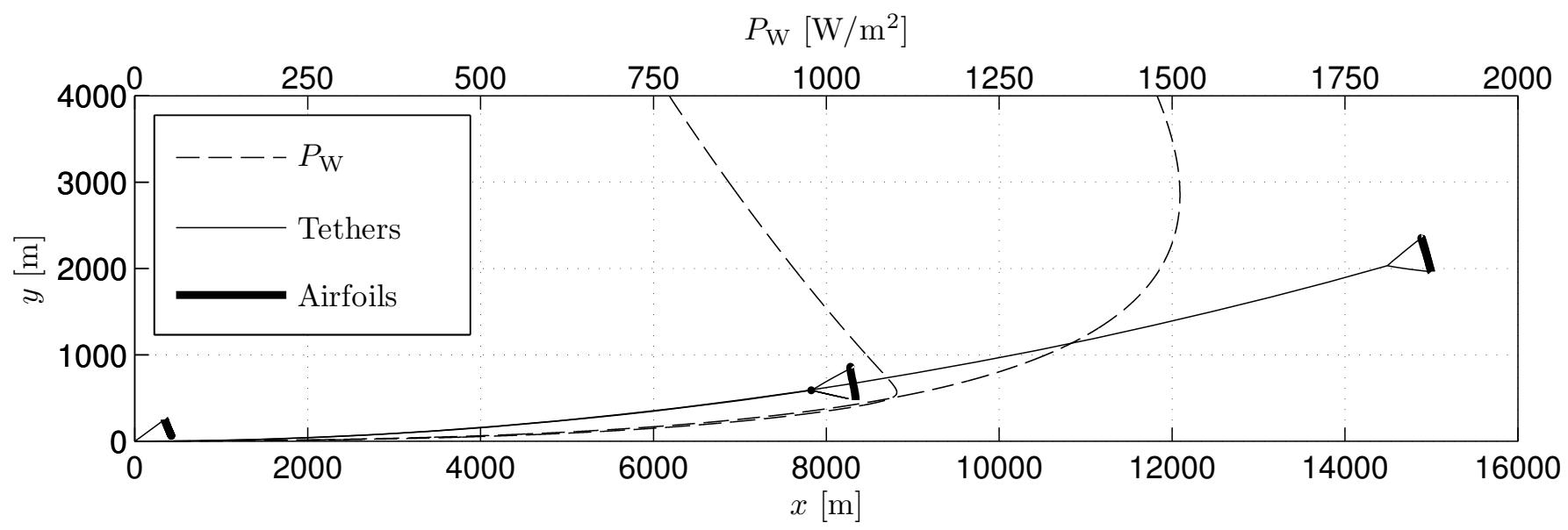

Fig. 6. Trajectory comparison between the single and dual airfoils for a total wing surface $S_{\text {tot }}=500 \mathrm{~m}^{2}$. The trajectories are shown as thick lines. The available wind power $P_{w}=\rho W_{\infty}^{3} / 2$ is plotted as a dashed line. For the dual airfoils, two wind profiles have been considered: a) the logarithmic profile (1) and b) the logarithmic profile saturated above $z=500 \mathrm{~m}$.

$500 \mathrm{~m}^{2}$, is displayed in Figure 6. It can be noted that the dual airfoils operate at much higher altitude, approaching the peak of the available wind power formula $(2850 \mathrm{~m})$, also displayed in Figure 6. The proposed wind shear model (1) is valid only in the atmospheric boundary layer, which is typically lower than $2000 \mathrm{~m}$. In this paper, the boundary layer was supposed to have an infinite thickness. The resulting optimal trajectories for the dual-kite systems reaches over $2000 \mathrm{~m}$, which strongly suggests that the optimal altitude is always at the top of the boundary layer, regardless of its thickness. Arguably, in boundary layers that are not developed to the top altitude of $2000 \mathrm{~m}$, the dual-airfoil system would loose some of its efficiency, and its advantage over the single-airfoil system would be reduced. In regard of these results, in practice, the optimization of a dual-airfoil AWE system should consider the average altitude of the boundary layer in the region of interest. As a term of comparison, a second scenario has been considered, where the wind profile saturates for $z>500 \mathrm{~m}$. The resulting trajectory and related available wind power are also displayed in Figure 6. In this second case, the trajectory is still in proximity of the peak of available wind power, which occurs at much lower altitude. For a total wing surface $S_{\text {tot }}=500 \mathrm{~m}^{2}$, also in the case of a saturated wind profile, the power extracted by the dual airfoils exceeds the one extracted by the single airfoil by the ratio $P_{\text {dual }}=1.54 P_{\text {single }}$.

The tether length obtained for the dual-kite system is arguably extremely large. It was observed, however, that the sensitivity of the power generation to the tether length is rather small, i.e. constraining the tether to smaller length does not result in a large power loss. Arguably, economical factors such as the material cost and the electrical resistance of a very long tether would yield a system with a shorter tether. In this paper, however, only the physics of the system were considered.

To check the precision of the collocation discretization, an optimization for the biggest system has been run with a refined collocation scheme having 4 times more collocation elements, resulting in an NLP with 47350 variables. No relevant difference has been noticed in the resulting trajectory, suggesting that the chosen collocation scheme is accurate enough.

The proposed scenario assumes that the airfoils do not modify the wind field. The development of an accurate model needs extensive studies. Future research will aim at investigating the impact of the presence of the airfoils on the wind field. Early results relying on simplified interaction models suggest that the dual airfoils would still extract more power than the single airfoil. Yet, a higher performance loss is observed for the dual-airfoil system.

Observe that the computed trajectories are only valid for the nominal case and in a real application wind perturbations and unmodeled dynamics will affect the performance of the system: this problem can be tackled within a robust optimization framework. The resulting NLP will, though, be considerably more complex than the proposed one. In the context of a real application, performance is also affected by the choice of the controller. Both investigations are out of the scope of this paper and are the subject of ongoing research.

\section{CONCLUSION \& FURTHER DEVELOPMENTS}

This paper has proposed a generic multiple-airfoil modeling procedure of minimal computational complexity, aimed for the optimization of power generation. This procedure can straightforwardly accomodate for 6-DOF airfoil models.

The proposed procedure has been applied to develop a large-scale model for the comparison of single vs. dual-airfoil systems so as to investigate which system is best suited, given the required average power output.

The results show that dual systems extract more power for all scales on the given scenario. Scaling up from small to large scales, the ratio of the power extracted by the dual airfoils vs. the one extracted by the single airfoil decreases.

Tether elasticity has been neglected and the airfoil model has been simplified for the sake of clarity of the presentation. Future research will focus on building a model database for both airfoils and tethers to be interfaced to the modeling procedure and optimization routines proposed. 
For a more accurate study, the interaction between the airfoils and the airmass should be included in the model. A Computational Fluid Dynamics (CFD) simulation is the object of ongoing research.

\section{ACKNOWLEDGMENTS}

The authors wish to thank Attila Kozma for his technical support and Reinhart Paelinck for the illustration in Figure 1. This research was supported by Research Council KUL: PFV/10/002 Optimization in Engineering Center OPTEC, GOA/10/09 MaNet and GOA/10/11 Global real- time optimal control of autonomous robots and mechatronic systems. Flemish Government: IOF/KP/SCORES4CHEM, FWO: PhD/postdoc grants and projects: G.0320.08 (convex MPC), G.0377.09 (Mechatronics MPC); IWT: PhD Grants, projects: SBO LeCoPro; Belgian Federal Science Policy Office: IUAP P7 (DYSCO, Dynamical systems, control and optimization, 2012-2017); EU: FP7-EMBOCON (ICT248940), FP7-SADCO ( MC ITN-264735), ERC ST HIGHWIND (259 166), Eurostars SMART, ACCM.

\section{REFERENCES}

[1] J. Andersson, J. Åkesson, and M. Diehl. CasADi - A symbolic package for automatic differentiation and optimal control. In S. Forth, P. Hovland, E. Phipps, J. Utke, and A. Walther, editors, Recent Advances in Algorithmic Differentiation, Lecture Notes in Computational Science and Engineering, Berlin, 2012. Springer.

[2] D.G. Andrews. An Introduction to ATMOSPHERE PHYSICS. Cambridge University Press, 2010.

[3] Bianchi, Fernando D. and Battista, Hernn de and Mantz, Ricardo J. Wind Turbine Control Systems. Springer, 2007.

[4] Lorenz T. Biegler. Nonlinear Programming. MOS-SIAM Series on Optimization. SIAM, 2010.

[5] E. A. Bossanyi. Further Load Reductions with Individual Pitch Control. Wind Energy, 8:481-485, 2005.

[6] S. Boyd and L. Vandenberghe. Convex Optimization. University Press, Cambridge, 2004.

[7] M. Canale, L. Fagiano, and M. Milanese. High Altitude Wind Energy Generation Using Controlled Power Kites. IEEE Transactons On Control Systems Technology, 18:168 - 180, 2010.

[8] Michael V. Cook. Flight Dynamics Principles. Elsevier Science, 2007.

[9] de Jalón, J. G. and Bayo, E. Kinematic and Dynamic Simulation of Multibody Systems: The Real-Time Challenge. Springer-Verlag, 1994.

[10] M. Diehl. Real-Time Optimization for Large Scale Nonlinear Processes. PhD thesis, Universität Heidelberg, 2001. http://www.ub.uniheidelberg.de/archiv/1659/.

[11] M. Diehl and B. Houska. Windenergienutzung mit schnell fliegenden Flugdrachen: eine Herausforderung für die Optimierung und Regelung - Wind Power via Fast Flying Kites: a Challenge for Optimization and Control. at-automatisierungstechnik, 57(10):525-533, 2009.

[12] L. Fagiano, M. Milanese, and D. Piga. High-Altitude Wind Power Generation. IEEE Transactions on Energy Conversion, 25:168-180, 2010.

[13] L. Fagiano, M. Milanese, and D. Piga. Optimization of Airborne Wind Energy Generators. International Jounal of Robust and Nonlinear Control, 2011.

[14] B. Houska and M. Diehl. Optimal control of towing kites. In Proceedings of the 45th IEEE Conference on Decision and Control, pages 2693-2697, San Diego, USA, 2006.

[15] B. Houska and M. Diehl. Optimal Control for Power Generating Kites. In Proc. 9th European Control Conference, pages 3560-3567, Kos, Greece,, 2007. (CD-ROM).

[16] J.H. Laks, L.Y. Pao, and A.D. Wright. Control of Wind Turbines: Past, Present, and Future. In American Control Conference, pages 2096-2103, 2009.

[17] M.L. Loyd. Crosswind Kite Power. Journal of Energy, 4(3):106-111, July 1980

[18] Makani Power. Makani Power Homepage. http://www.makanipower.com/.

[19] Manwell, J. F., McGowan, J. G. and Rogers, A. L. Wind Energy Explained: Theory, Design and Application, Second Edition. 2009.

[20] Pamadi. Performance, Stability, Dynamics, and Control of Airplanes. American Institute of Aeronautics and Astronautics, Inc., 2003.

[21] P. Payne and Ch. McCutchen. Self-Erecting Windmill. United States Patent 3987987, Oct. 261976.

[22] A.R. Podgaets and W.J. Ockels. Flight control and stability of a multiple kites tethered system. In Proceedings of Renewable Energy Conference, 2006.
[23] A. Wächter and L.T. Biegler. On the Implementation of a Primal-Dual Interior Point Filter Line Search Algorithm for Large-Scale Nonlinear Programming. Mathematical Programming, 106(1):25-57, 2006.

[24] P. Williams, B. Lansdorp, and W. Ockels. Optimal Crosswind Towing and Power Generation with Tethered Kites. Journal of Guidance, Control, and Dynamics, 31(1):81-92, January-February 2008.

[25] Williams, P. and Lansdorp, B. and Ockels, W.J. Modeling of Optimal Power Generation using Multiple Kites. In AIAA Modelling and Simulation Technologies Conference and Exhibit, Honolulu, Hawaii, USA, 2008. 